\title{
Noniterative Algorithms for Electrical Resistivity Imaging Applied to Subsurface Local Anomalies
}

\author{
Manel Gasulla and Ramon Pallàs-Areny, Fellow, IEEE
}

\begin{abstract}
In this paper, we compare five noniterative (one-step) algorithms for two-dimensional electrical resistivity imaging applied to the location of subsurface local anomalies. Here, we analyze the performance of two backprojection algorithms and three algorithms based on a least-squares criterion. These five algorithms can also be adapted for process and medical tomography. Algorithm performance is first assessed from synthetic data derived from an analytical solution. We show that least-squares-based algorithms outperform backprojection algorithms in all situations considered. One of the least-squares algorithms was further validated with experimental measurements involving spherical objects immersed into a water tank. Data were obtained using a 16-electrode linear array and a computer-controlled data-acquisition system. A reference measurement before immersing the objects into the water tank reduced errors in the reconstructed image attributable to the uncertain electrode position and the finite dimensions of the tank. Images deteriorated for deeper objects, but neglecting measurements with the smallest signal-to-noise ratio improved the results.
\end{abstract}

Index Terms-Electrical resistivity tomography, impedance imaging, noniterative image reconstruction, subsurface imaging.

\section{INTRODUCTION}

$\mathbf{E}$ LECTRICAL IMPEDANCE TOMOGRAPHY (EIT) has been proposed for several applications in fields ranging from industry to medicine and subsurface investigation. EIT aims to image the electrical impedance distribution within a volume, e.g., a process vessel, a patient's body, or the subsoil, using noninvasive techniques. Normally, an array of electrodes is used where current is injected using a pair of electrodes and the resulting voltage drops are measured with the remaining electrode pairs. In industrial and medical applications, the electrode array often surrounds the body, whereas in subsurface imaging electrodes are located on the surface. In industrial and medical applications, the resistance [electrical resistance tomography (ERT)] or capacitance [electrical capacitance tomography (ECT)] are of interest, whereas subsurface imaging usually investigates resistivity distributions.

Image reconstruction methods can be implemented as either noniterative or iterative algorithms. Iterative methods calculate the sensitivity matrix and surface potentials at each iteration using a computer model (finite elements, difference elements), which is computation-intensive. This problem worsens for

Manuscript received February 20, 2004; revised February 11, 2005. This work was supported by the Spanish Ministry of Science and Technology under Project TAP99-0742. The associate editor coordinating the review of this paper and approving it for publication was Prof. Paul P. L. Regtien.

The authors are with the Escola Politècnica Superior de Castelldefels, Universitat Politècnica de Catalunya (UPC), 08860 Castelldefels, Barcelona, Spain (e-mail: manel.gasulla@upc.edu; ramon.pallas@upc.edu).

Digital Object Identifier 10.1109/JSEN.2005.858970 three-dimensional (3-D) imaging because this involves more data and parameters. Several authors have proposed different techniques to reduce computation time in subsurface imaging, as in [1] and [2], but convergence problems remain that may lead to the wrong solution. Noniterative algorithms (also called "one-step") can be considered a particular case of iterative algorithms where only the first iteration is performed. If, in addition, the starting model is a homogeneous body, the computation time is largely reduced [3]. The drawback is a lower "quality" of the obtained images.

To achieve a fast image reconstruction, algorithms in process and medical impedance tomography use to be noniterative and based on backprojecting the voltages measured with a circular electrode array. Planar (rectangular and linear) electrode arrays have also been proposed [4]-[6], but they are less common. On the other hand, subsurface imaging does not normally involve any rapidly changing media, and, therefore, image reconstruction time is not critical. Hence, iterative algorithms are normally applied. In addition, least-squares methods are preferred although some backprojection algorithms derived from medical imaging have also been proposed [7], [8].

In contrast to the normal practice in subsurface imaging, here we propose to use noniterative algorithms applied to the location of subsurface local anomalies. Some previous work [9], [10] has already shown the feasibility of this approach. We analyze the performance of two backprojection algorithms and three algorithms based on a least-squares criterion. The paper first reviews the theoretical basis of the algorithms and their differences and then compares images obtained from synthetic and experimental data.

\section{THEORETICAL BACKGROUND OF THE RECONSTRUCTION ALGORITHMS}

\section{A. Sensitivity Theorem and Matrix}

To solve an inverse problem, the (theoretical) model that relates the data (measurements at the boundary) to the searched parameters (conductivity, resistivity, permittivity) must first be determined. The sensitivity theorem mathematically relates model and measurements [11], [12]. Consider a volume $V$ of conductivity $\sigma(x, y, z)$ bounded by the surface $S$ (Fig. 1), which does not include any internal electrical source. If a current $I_{\phi}$ from A to B yields a potential distribution $\phi(x, y, z)$, and a current $I_{\psi}$ from $\mathrm{M}$ to $\mathrm{N}$ yields a potential distribution $\psi(x, y, z)$, then

$$
z=\frac{\psi_{\mathrm{AB}}}{I_{\psi}}=\frac{\phi_{\mathrm{MN}}}{I_{\phi}}=\int_{V} \sigma \frac{\nabla \phi(\sigma)}{I_{\phi}} \frac{\nabla \psi(\sigma)}{I_{\psi}} d V
$$




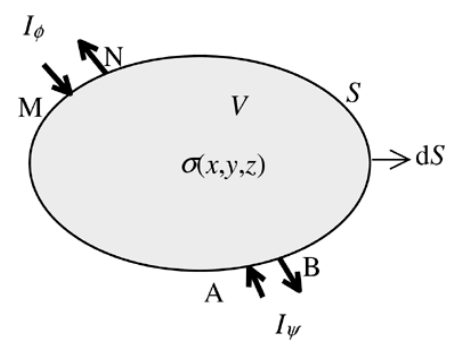

Fig. 1. Volume of conductivity $\sigma(x, y, z)$.

where $\psi_{\mathrm{AB}}$ and $\phi_{\mathrm{MN}}$ are the potential differences between, respectively, electrodes pairs A-B and $\mathrm{M}-\mathrm{N}$, and $z$ is defined as the mutual impedance between electrode pairs. Therefore, the relations between boundary (surface) potentials and the internal conductivity distribution are reciprocal, meaning that the mutual impedance measured when injecting current through a first electrode pair and measuring voltage using a second electrode pair, is the same obtained when using the second pair for current injection and the first pair for voltage detection. This property can be used to obtain redundant measurements able to reveal possible measurement errors. The problem is nonlinear because $\nabla \phi$ and $\nabla \psi$ depend on the conductivity $\sigma$, so that it is impossible to estimate the conductivity distribution (inverse problem) from (1). When the conductivity distribution changes from $\sigma^{0}(x, y, z)$ to $\sigma^{0}(x, y, z)+\Delta \sigma(x, y, z)$, the mutual impedance changes by

$$
\Delta z=-\int_{V} \Delta \sigma \frac{\nabla \phi\left(\sigma^{0}\right)}{I_{\phi}} \frac{\nabla \psi\left(\sigma^{0}+\Delta \sigma\right)}{I_{\psi}} d V
$$

By expanding $\nabla \psi\left(\sigma^{0}+\Delta \sigma\right)$ with respect to $\Delta \sigma$, (2) leads to [13]

$$
\Delta z=-\int_{V} \Delta \sigma \frac{\nabla \phi\left(\sigma^{0}\right)}{I_{\phi}} \frac{\nabla \psi\left(\sigma^{0}\right)}{I_{\psi}} d V+O\left((\Delta \sigma)^{2}\right)
$$

where $O\left((\Delta \sigma)^{2}\right)$ indicates the higher order terms with respect to $\Delta \sigma$. If $\Delta \sigma$ is small enough, $O\left((\Delta \sigma)^{2}\right)$ can be disregarded. In (3), $\nabla \phi$ and $\nabla \psi$ are independent from $\Delta \sigma$ and $\Delta z$ linearly depends on $\Delta \sigma$. To obtain $\Delta \sigma$ from (3), the volume $V$ is divided in small volumes of constant conductivity. Fig. 2 shows a two-dimensional $(2-D)$ example with $80(16 \times 5)$ elements (pixels) corresponding to the vertical section just under the electrodes. In 3-D problems, several contiguous vertical sections of the subsurface are discretized.

The sensitivity is equally calculated for 2-D and 3-D problems. Electric fields in 2-D problems are calculated from the analytical 3-D solution, so that no errors are introduced. Neglecting $O\left((\Delta \sigma)^{2}\right)$, (3) can be expressed as

$$
\begin{aligned}
\Delta z_{i} & =\sum_{j=1}^{Q} \Delta \sigma_{j} \int_{\operatorname{pixel} j} \boldsymbol{L}_{i}(\phi) \boldsymbol{L}_{i}(\psi) d v_{j} \\
i & =1 \ldots P
\end{aligned}
$$

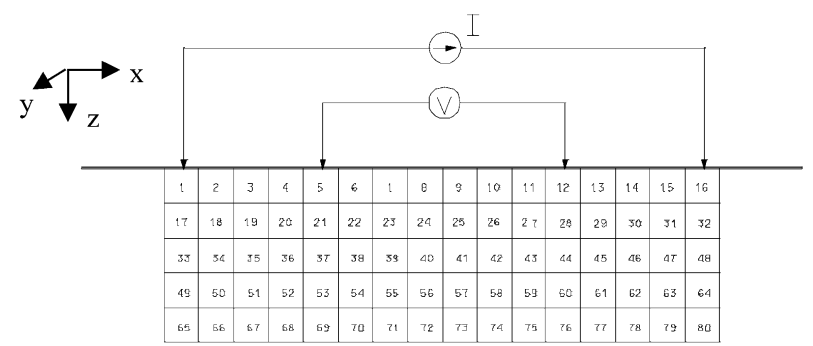

Fig. 2. Two-dimensional division of a volume in $80(16 \times 5)$ elements (pixels) of constant conductivity.

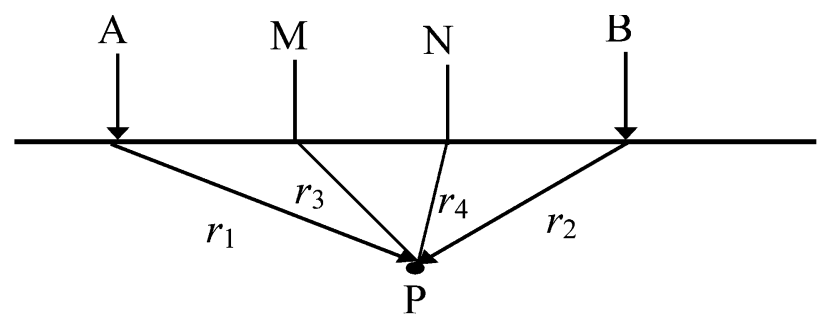

Fig. 3. Distances of electrodes A, B, M, N, to a point P.

where $Q$ is the number of pixels, $P$ is the number of measurements, and the lead vectors for each measurement $i$ are defined as

$$
\begin{aligned}
\boldsymbol{L}_{i}(\phi) & =\frac{\nabla \phi\left(\sigma^{0}\right)}{I_{\phi}} \\
\boldsymbol{L}_{i}(\psi) & =\frac{\nabla \psi\left(\sigma^{0}\right)}{I_{\psi}} .
\end{aligned}
$$

Each new measurement accounts for one position of the four electrodes A, B, M, and N. Using matrix notation, (4) can be written as

$$
\Delta Z=S \Delta \Sigma
$$

where $\Delta \boldsymbol{Z}$ and $\Delta \boldsymbol{\Sigma}$ are vectors whose respective dimensions are $P \times 1$ and $Q \times 1$, and $S$ is the sensitivity matrix, whose dimension is $P \times Q$. The elements of $S$ are

$$
s_{i j}=-\int_{\text {pixel } j} L_{i}(\phi) L_{i}(\psi) d v_{j}
$$

and each of them describes the sensitivity of pixel $j$ to the measurement $i$. If the initial conductivity distribution $\left(\sigma^{0}\right)$ is homogeneous, the lead vectors in (5) are

$$
\begin{aligned}
& \boldsymbol{L}_{i}(\phi)=\frac{\rho}{2 \pi}\left(\frac{\vec{r}_{1}}{\left|\vec{r}_{1}\right|^{3}}-\frac{\vec{r}_{2}}{\left|\vec{r}_{2}\right|^{3}}\right) \\
& \boldsymbol{L}_{i}(\psi)=\frac{\rho}{2 \pi}\left(\frac{\vec{r}_{3}}{\left|\vec{r}_{3}\right|^{3}}-\frac{\vec{r}_{4}}{\left|\vec{r}_{4}\right|^{3}}\right)
\end{aligned}
$$

where $r_{1}$ to $r_{4}$ are the respective distances from electrodes A, B, M, and N to the point P (Fig. 3). Equation (8) applies to both 2-D and 3-D problems. In problems with circular geometry, (8) is normally calculated by numerical methods under the assumption that the geometry is truly 2-D, both for the body and for the injected currents (i.e., no current flows outside the target plane). This simplifying assumption can lead to unacceptable results 
when applying inverse algorithms to obtain the volume conductivity distribution.

To calculate the elements of the sensitivity matrix, we substitute (8) in (7) and numerically integrate in every pixel using the Gauss method [3]. The sensitivity matrix can be calculated and saved before applying the inversion algorithm in order to speed up the calculation process. Therefore, the real conductivity can be estimated by solving (6) and updating the homogeneous conductivity as

$$
\boldsymbol{\Sigma}^{\mathrm{est}}=\boldsymbol{\Sigma}^{0}+\Delta \boldsymbol{\Sigma}
$$

where $\boldsymbol{\Sigma}^{\text {est }}$ is the vector of estimated conductivities, $\boldsymbol{\Sigma}^{0}$ is the initial homogeneous conductivity, and $\Delta \Sigma$ is the change in conductivity obtained from (6). Image reconstruction algorithms differ mainly on how to calculate $\Delta \boldsymbol{\Sigma}$ from (6). Next sections describe this process for least-squares and backprojection algorithms.

\section{B. Least-Squares Algorithms}

If $S$ were a square matrix and its inverse existed, (6) could be solved as

$$
\Delta \Sigma=S^{-1} \Delta Z
$$

where $\Delta Z$ is the measured change in mutual impedance from the homogeneous to the actual conductivity distribution. If $P>Q$, the equation system is overdetermined and there is no unique solution. Defining a residual error $e$ and its quadratic (or Euclidian) norm $E$ as

$$
\begin{gathered}
\boldsymbol{e}=\Delta \boldsymbol{Z}-S \Delta \boldsymbol{\Sigma} \\
E=e^{T} e=(\Delta \boldsymbol{Z}-S \Delta \boldsymbol{\Sigma})^{T}(\Delta \boldsymbol{Z}-S \Delta \boldsymbol{\Sigma})
\end{gathered}
$$

we obtain a solution that minimizes $E$, as follows:

$$
\Delta \boldsymbol{\Sigma}=\left(S^{T} S\right)^{-1} S^{T} \Delta \boldsymbol{Z}
$$

If $P<Q$, the equation system is underdetermined and there are infinite solutions. A unique solution can be obtained by incorporating some a priori information, for example by minimizing the quadratic norm of the solution, $(\Delta \boldsymbol{\Sigma})^{T}(\Delta \boldsymbol{\Sigma})$. In this case, the solution is

$$
\Delta \Sigma=S^{T}\left(S S^{T}\right)^{-1} \Delta Z
$$

Because the inverse problem is ill posed, $S^{-1}, S^{T} S$, or $S S^{T}$ are singular or quasisingular. This implies that small errors in the data (measurements) may lead to unacceptable solutions [14]. This can be better understood by a singular-value decomposition (SVD) of $S$, to obtain

$$
S=U \Lambda V^{T}
$$

where $U(P \times P)$ and $V(Q \times Q)$ are orthogonal matrices, $\Lambda=$ $\operatorname{diag}\left(\lambda_{1}, \ldots, \lambda_{n}\right) \in \Re^{P \times Q}, n=\min \{P, Q\}$, and the singular values satisfy the condition $\lambda_{1} \geq \lambda_{2} \geq \cdots \geq \lambda_{n} \geq 0$ [15]. The matrix condition number is defined as $\lambda_{1} / \lambda_{n}$. The rank $r$ of $S$ is the number of singular values greater than zero. The solution to (6) can then be obtained as [16]

$$
\Delta \Sigma=V \Lambda^{-1} U^{T} \Delta Z
$$

In discrete ill-posed problems, the condition number of $S$ is large (i.e., $S$ is ill-conditioned), and the singular values of $S$

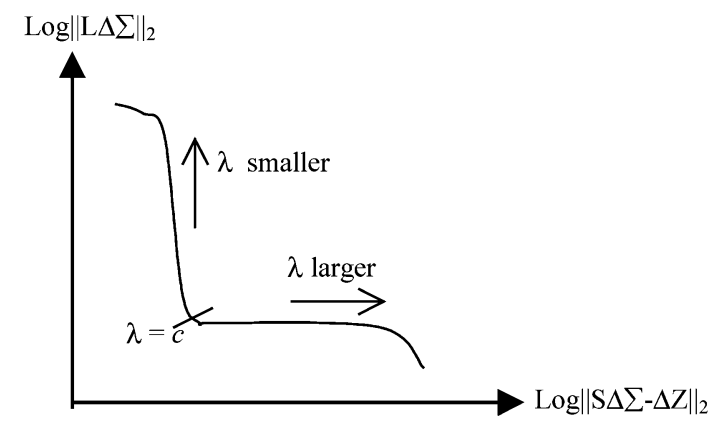

Fig. 4. Generic form of the L-curve.

gradually decay to zero [17]. Therefore, the smaller singular values (whose inverse will be a very large number) will greatly amplify data errors in the solution.

To obtain a stable and suitable solution to (6), it is necessary to add some a priori information about the desired solution, that is, we must regularize the problem. A possible regularization method is to calculate (15) using only the largest singular values, which are less susceptible to data errors, i.e., the smaller singular values are equated to zero. The rank $r$ of $S$ in (14) then reduces to the number of singular values different from zero. This method, called TSVD (truncated SVD) [17], has been widely used in medical tomography [13], [18]-[22]. Yang and Peng [23] review several reconstruction algorithms, including the TSVD, for ECT applied to industrial processes.

The Tikhonov regularization method [24] has been used by several authors. Here, the solution is obtained by minimizing a weighted combination of the residual error norm and a seminorm of the solution as

$$
\Delta \boldsymbol{\Sigma}=\arg \min \left\{\|S \Delta \boldsymbol{\Sigma}-\Delta Z\|_{2}^{2}+\lambda\|L \Delta \boldsymbol{\Sigma}\|_{2}^{2}\right\}
$$

where $\lambda$ is a regularization parameter and $L$ can be either the identity matrix of order $Q \times Q$ or a discrete approximation $J \times Q$ of a derivative operator of order $Q-J$ [17]. The first term in (16) measures the prediction error whereas the second term accounts for prior information in the solution [25]. The success of the method depends on selecting a convenient value for $\lambda$. A large $\lambda$ minimizes the effects of data errors on the solution but does not minimize the prediction error, and, hence, the solution can be a bad estimate of the true solution. A small $\lambda$ minimizes the prediction error but increases the influence of data errors, thus leading to unacceptable results.

Usually, $\lambda$ is empirically found [3], [26]. Hansen and O'Leary [27], [28] describe the L-curve method to find $\lambda$ automatically and show that this method is more robust than alternative methods. Fig. 4 outlines the basis of the L-curve method. The semi-norm $\|L \Delta \Sigma\|_{2}$ of the regularized solution is plotted against the norm of the prediction error $\|S \Delta \Sigma-\Delta Z\|_{2}$. In logarithmic scales, the curve normally has an L shape [28]. The intersection point $c$ between the vertical and horizontal lines of the curve is often selected as the regularization parameter $(\lambda=c)$. Hansen [17] provides a MATLAB routine based on determining the point with maximal curvature. The solution to (16) is

$$
\Delta \boldsymbol{\Sigma}=\left(S^{T} S+\lambda L^{T} L\right)^{-1} S^{T} \Delta \boldsymbol{Z}
$$


The smoothness-constrained least-squares method, also called Occam's inversion, aims to obtaining a smooth solution by using for $L$ a discrete approximation of a first- or second-derivative operator. Several authors use this technique to obtain 2-D ([3], [26], [29], [30]) or 3-D ([1], [31]) subsurface images from resistance data. Another usual alternative for $L$ is the identity matrix ( $L=I)$, in which case, the solution is

$$
\Delta \boldsymbol{\Sigma}=\left(S^{T} S+\lambda I\right)^{-1} S^{T} \Delta \boldsymbol{Z}
$$

This method has received several different names, such as damped least-squares, constrained least-squares, MarquardtLevenberg [14], and standard Tikhonov regularization [23]. Breckon and Pidock analyzed it for medical applications [32]. Hua et al. [25] compared three image-reconstruction algorithms based on (17), one with $L=I$ (Marquardt-Levenberg algorithm), and two with $L$ equal to, respectively, the first- and the second-derivative operator (Occam's method). They used synthetic data with added random noise, and concluded that the Occam's method performs a spatial low-pass filtering and gives the best results. Loke and Dahlin [33] achieved similar conclusions for geophysical applications. However, Gasulla [9] showed that the Marquardt-Levenverg method can yield better images from local underground objects.

Lines and Treitel [14] showed that (18) can be expressed as

$$
\Delta \boldsymbol{\Sigma}=V \operatorname{diag}\left(\frac{\lambda_{j}}{\lambda_{j}^{2}+\lambda}\right) U^{T} \Delta \boldsymbol{Z}
$$

which relates the Marquardt formulation to the SVD formulation (15).

\section{Backprojection Algorithms}

The problems arising when trying to solve (6) by using least-squares methods, fostered the search of alternatives. One method that has become widely used in medical EIT is the linear backprojection method, analogous to the filtered backprojection technique used in X-ray computerized tomography. Kotre [4] proposed the following algorithm:

$$
\frac{\Delta \sigma_{j}}{\sigma_{j}^{o}}=-k \frac{\sum_{i=1}^{P} s_{i j} \frac{\Delta z_{i}}{z_{i}^{o}}}{\sum_{i=1}^{P} s_{i j}} j=1 \ldots Q
$$

where $k$ is an empirically selected amplification factor and it is assumed that conductivity changes are small. The algorithm, which we call total backprojection, can be expressed in matrix notation as

$$
\Delta \boldsymbol{\Sigma}_{r}=W S^{T} \Delta Z_{r}
$$

where $\Delta \boldsymbol{\Sigma}_{r}$ and $\Delta Z_{r}$ are normalized vectors and $W$ is a weighted diagonal matrix whose elements are

$$
w_{j j}=-\frac{k}{\sum_{i=1}^{P} s_{i j}} j=1 \ldots Q .
$$

This algorithm has been proposed for medical applications [5] and to detect abandoned landmines [34]. In fact, the algorithm is similar to the gradient or steepest descent method and could be obtained from (18) with $\lambda$ tending to infinite. As already

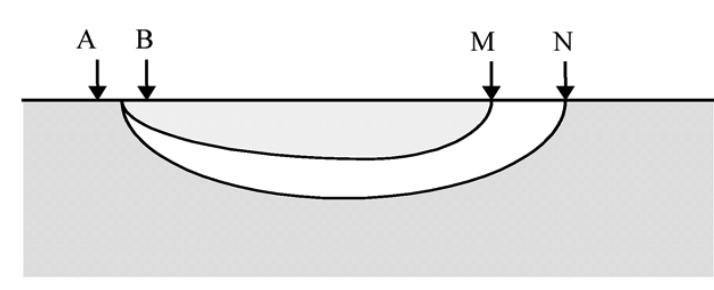

Fig. 5. Equipotential lines defined by electrodes $\mathrm{M}$ and $\mathrm{N}$.

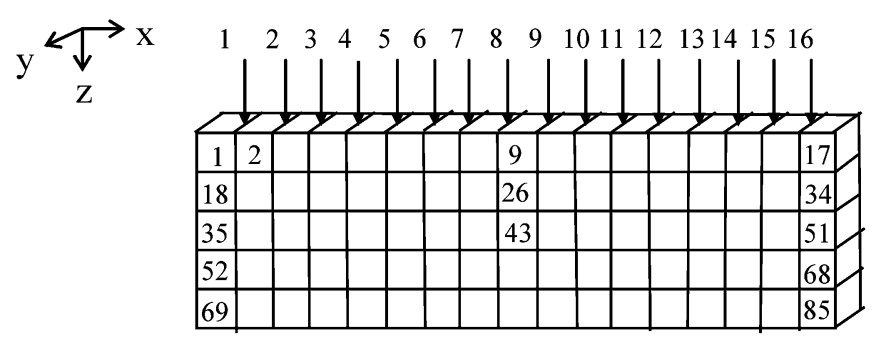

Fig. 6. Two-dimensional division of the subsoil in 85 voxels $(17 \times 5)$ corresponding to a vertical slice just under the 16 equally spaced electrode array.

mentioned, a large $\lambda$ yields a bad estimate of the true solution. Nevertheless, this algorithm (and similar ones) has been widely applied to on-line image reconstruction [23], mainly because of its simplicity.

A variation of (21) supposes that a change in the voltage difference measured between electrodes $\mathrm{M}-\mathrm{N}$ is only attributable to the change in conductivity in the zone delimited by the equipotential lines leaving from the measurement electrodes (Fig. 5). The conductivity can be estimated by (21) using as sensitivity coefficients

$s_{i j}= \begin{cases}s_{i j}, & \text { if the pixel is between equipotential lines } \\ 0, & \text { otherwise. }\end{cases}$

We call this method equipotential backprojection. Noel and $\mathrm{Xu}$ [7] proposed a similar method for geophysical prospecting where the sensitivity coefficients were additionally weighted by the percentage of the pixel falling between the equipotential lines. Tsourlos et al. [35] proposed an iterative solution. Backprojection between equipotential lines was first proposed by Barber et al. [36] for medical impedance tomography and yielded in vivo images of tissue resistivity [37]. They called their technique applied potential tomography and used some geometrically calculated weights. Image quality was improved by filtering in the space-frequency domain. Later on, this method was extended to linear electrode arrays [38].

\section{IMAGES FROM SyNTHETIC DATA}

In this section, we compare five different image reconstruction algorithms applied to local anomalies: TSVD, Marquardt, Occam, total backprojection, and equipotential backprojection. In the Occam's method, we use a discrete approximation of the second-derivative operator for $L$ [26]. The images are 2-D but the algorithms can be easily extended to obtain 3-D images [9]. The comparison is made using simulated data. Simulated data are often obtained by numerical methods. Here we have obtained the data from an analytical solution for a subsurface 
a)
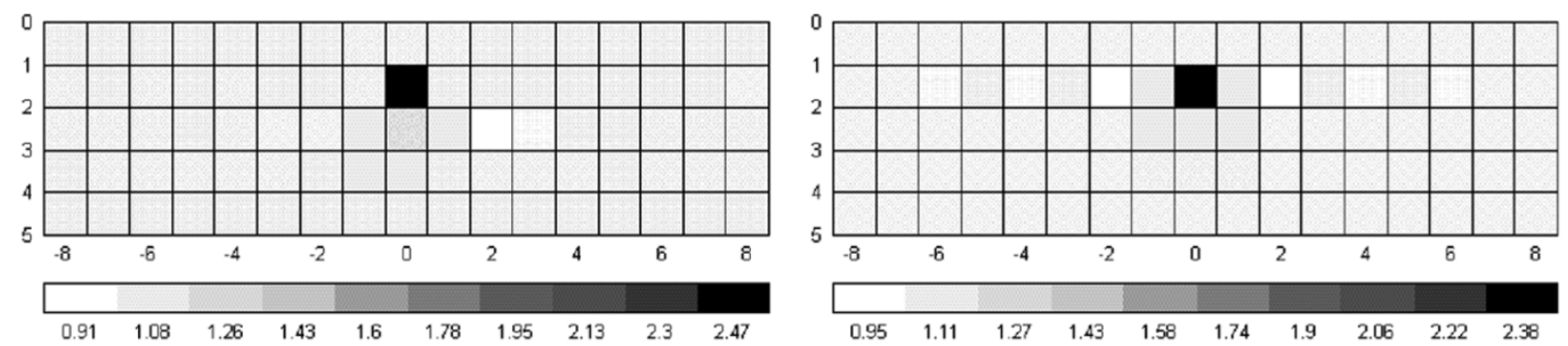

b)
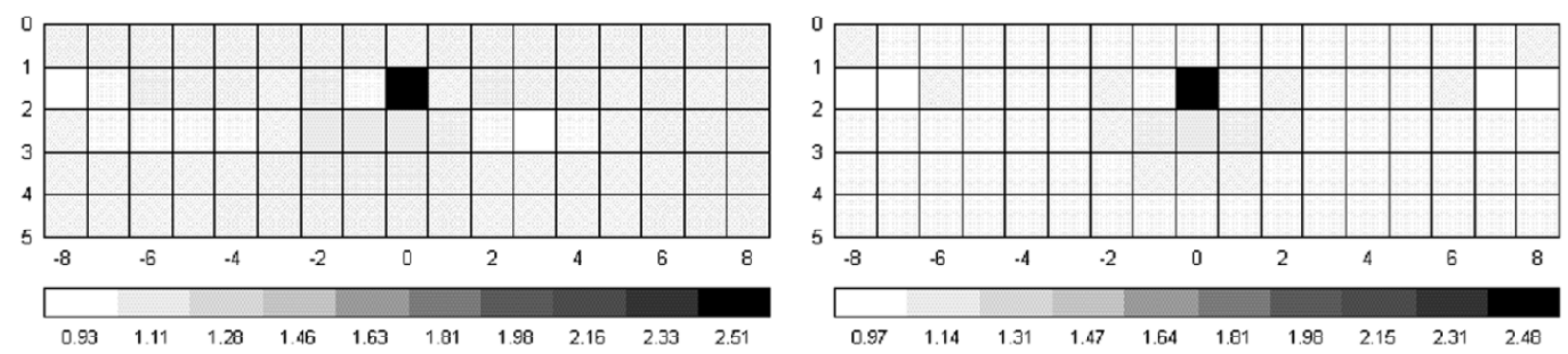

c)
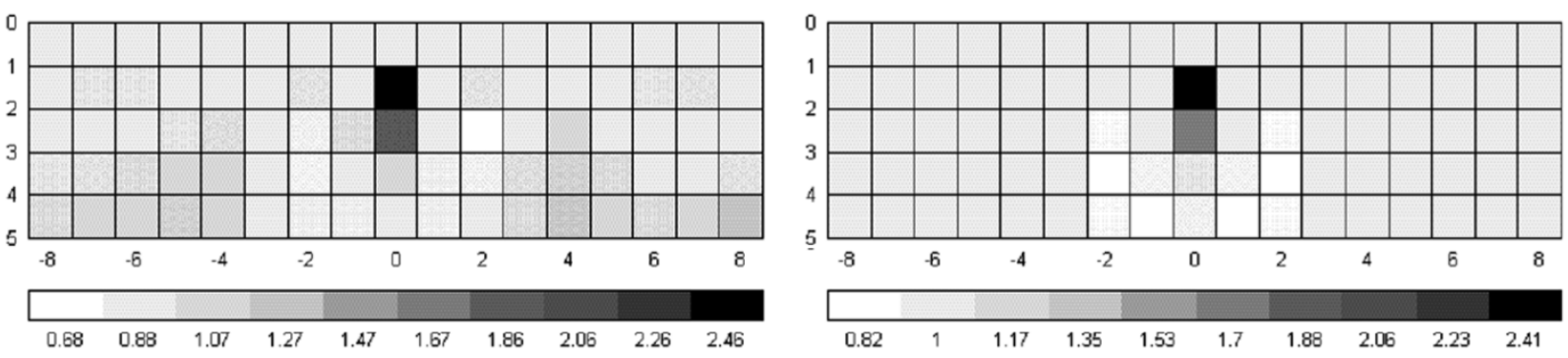

d)
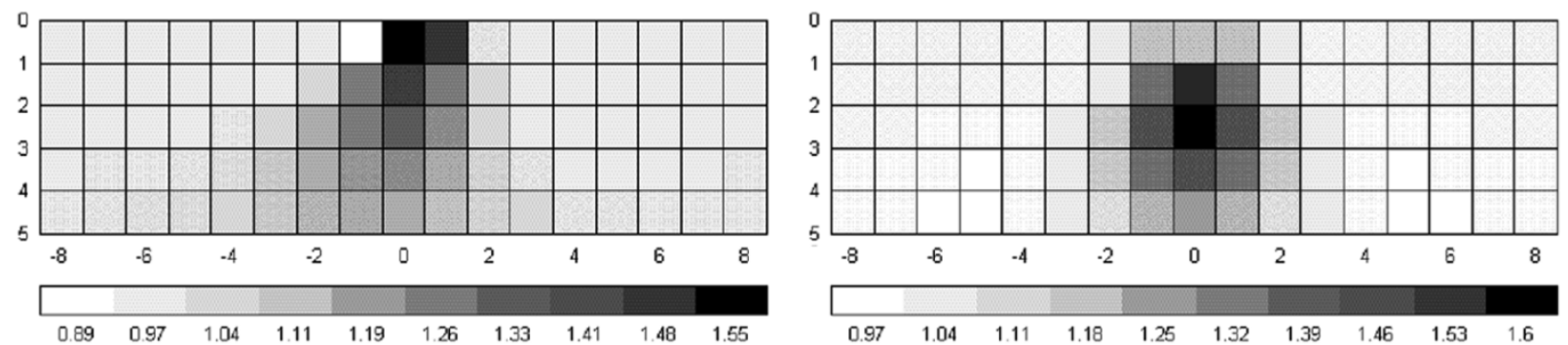

e)
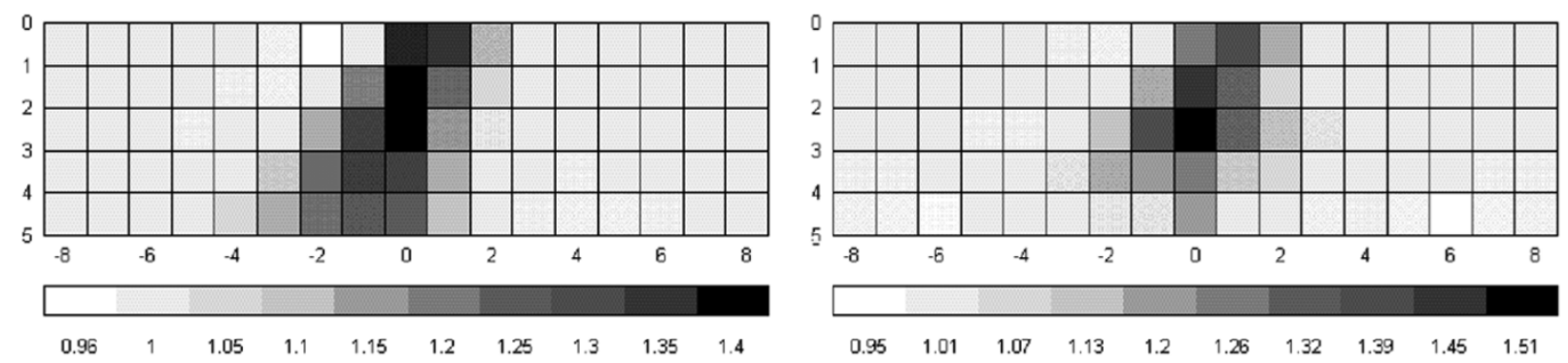

Fig. 7. Two-dimensional images obtained from different algorithms applied to synthetic data for (left) the Schlumberger and (right) dipole-dipole electrode arrays, for a perfectly conducting sphere of 0.5 unit radius placed at $x=y=0, z=1.5$. The 2-D model consists of 85 voxels $(17 \times 5)$ of one-unit side dimensions. (a) Marquardt $\left(\lambda=1.50 \times 10^{-8} ; 1.83 \times 10^{-8}\right)$. (b) TSVD $(r=35)$. (c) Occam $\left(\lambda=1.51 \times 10^{-9} ; 2.21 \times 10^{-9}\right)$. (d) Total backprojection. (e) Equipotential backprojection.

sphere immersed in a homogeneous ground. This yields, in contrast with numerical methods, a fast, accurate and inexpensive set of data. Section V includes images from experimental data.
To solve (6), we need $S$ and $\Delta \boldsymbol{Z}=\boldsymbol{Z}-\boldsymbol{Z}^{0}, \boldsymbol{Z}$, and $\boldsymbol{Z}^{0}$ being, respectively, the mutual impedances corresponding to the actual and the homogeneous conductivity distribution. In subsurface 
a)

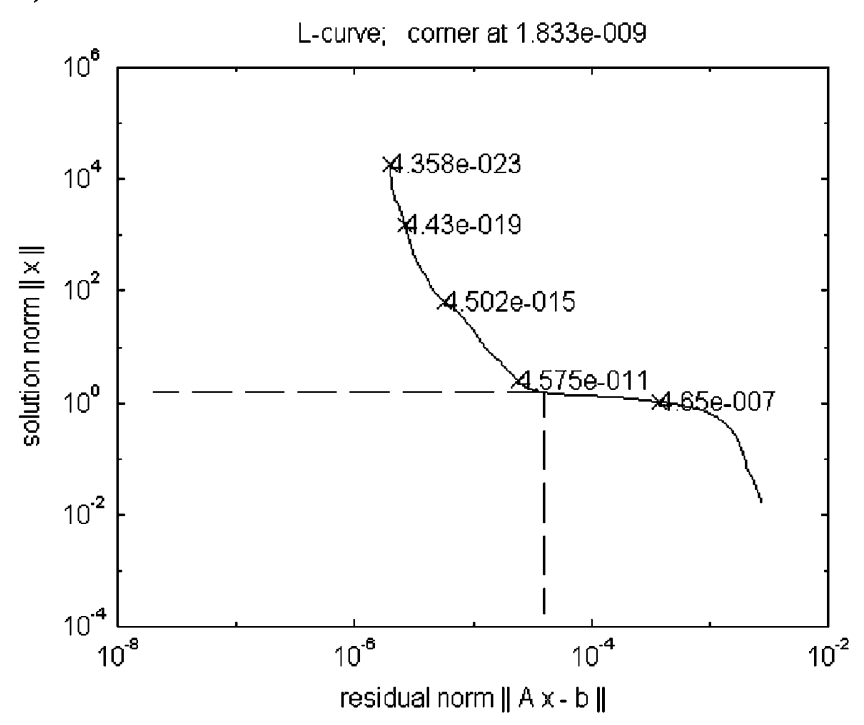

c)

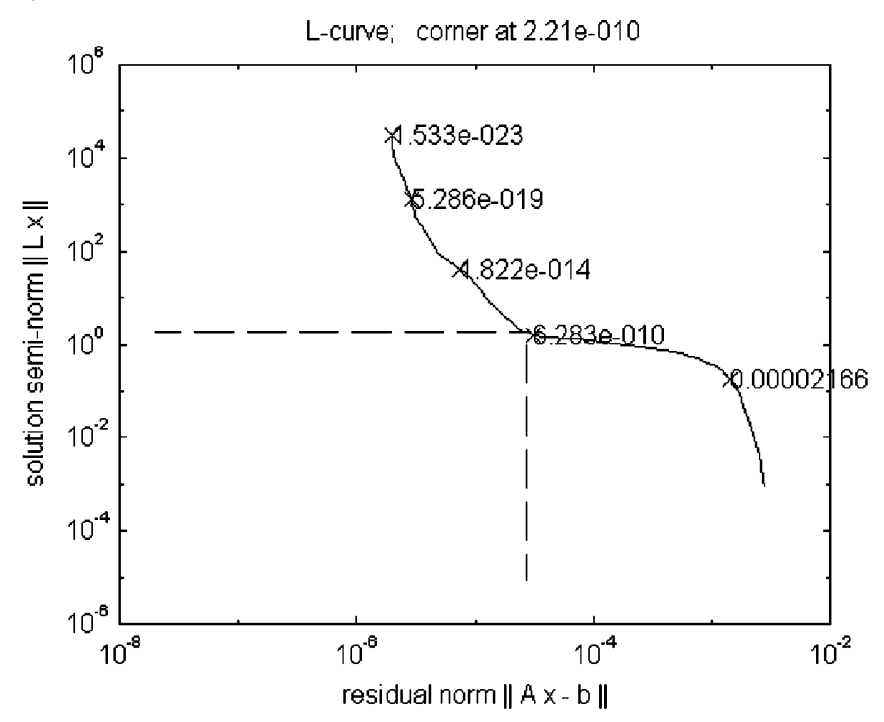

b)

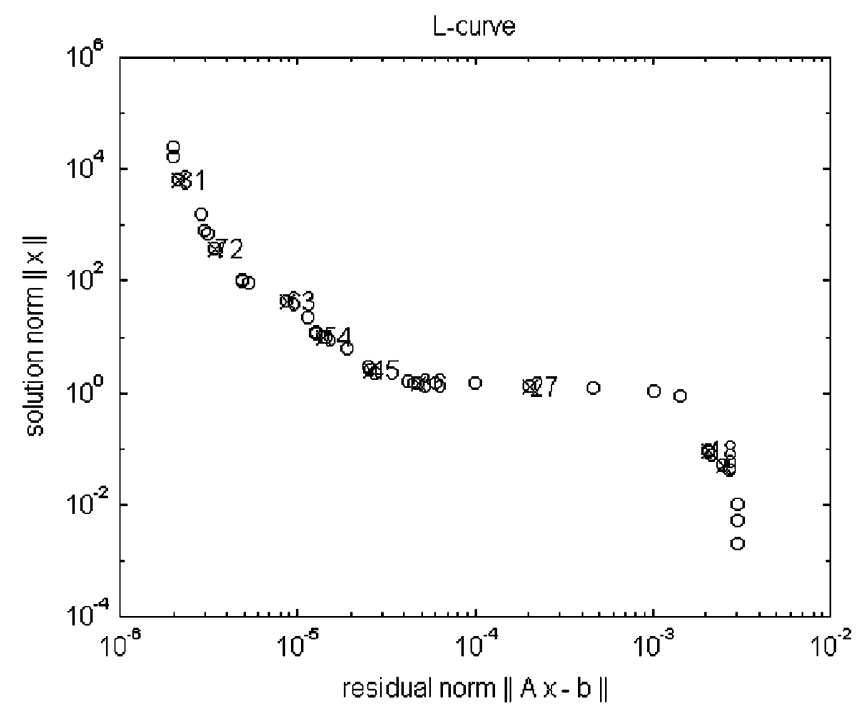

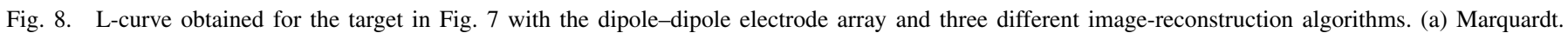
(b) TSVD. (c) Occam.

imaging, $Z^{0}$ can be straightforwardly calculated [39]. $Z$ can be analytically calculated for simple objects such as a sphere immersed in a homogeneous medium of different conductivity [9], [39]. Therefore, the five algorithms were compared in applications aiming to find sphere-like local objects, as opposed to imaging distributed conductivity changes. The sensitivity matrix was calculated for a homogeneous medium by using the procedure described in Section II-A.

We used two different electrode arrays: a modified Schlumberger array and a dipole-dipole array. Each array included 16 uniformly spaced electrodes (Fig. 6). The current-injecting electrode pairs for the modified Schlumberger array were $(1,16)$, $(2,16)$, and so on, up to $(13,16)$. For each current pair, we measured the voltage difference between successive pairs of internal adjacent electrodes, which yielded 91 measurements. True, 16 electrodes can yield up to 104 independent measurements [7]; the remaining 13 measurements result from injecting by electrode pair $(1,15)$ and detecting the voltage difference between the remaining pairs of internal adjacent electrodes (12 measurements), and injecting by electrode pair $(3,16)$ and detecting the voltage difference between the electrode pair $(1,2)$. The dipole-dipole array is equivalent to the adjacent configuration used in process and medical tomography. The current-injecting electrode pairs were $(1,2),(2,3)$, and so on up to $(13,14)$. For each current pair, we measured the voltage difference between successive pairs of adjacent electrodes located at the right side of the current-injecting electrode pair, which yielded 91 measurements. The remaining 13 measurements result from injecting by electrode pair $(16,1)$ and detecting the voltage difference between the remaining pairs of internal adjacent electrodes.

To calculate the regularization parameter in the Marquardt and Occam's algorithms, we used the MATLAB routines implemented in [17]. We found that a suitable value for $\lambda$ was from 10 to 100 times the value $c$ obtained by the method of the L-curve. In the TSVD method, the rank $r$ of $S$ in (14) was determined from the discrete L-curve. In the backprojection algorithms, we 
a)

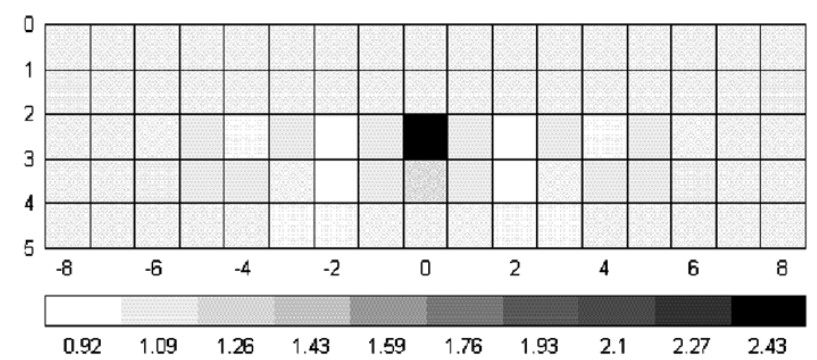

c)

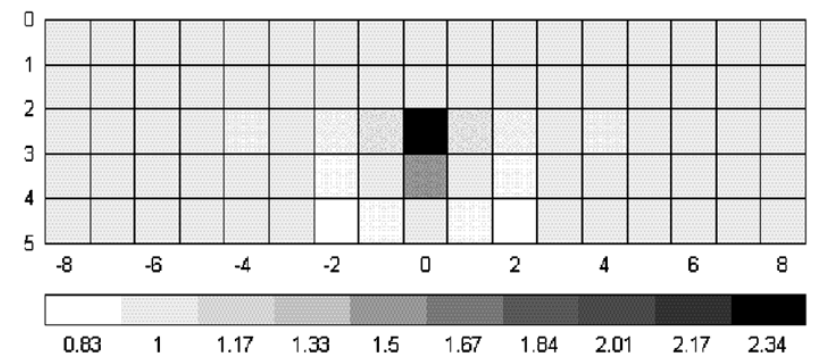

e)

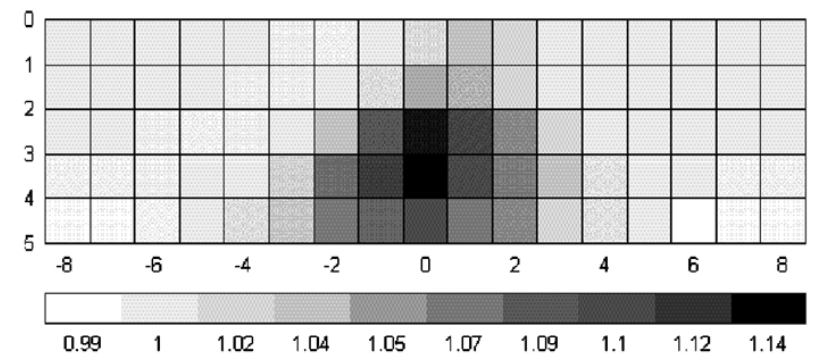

b)

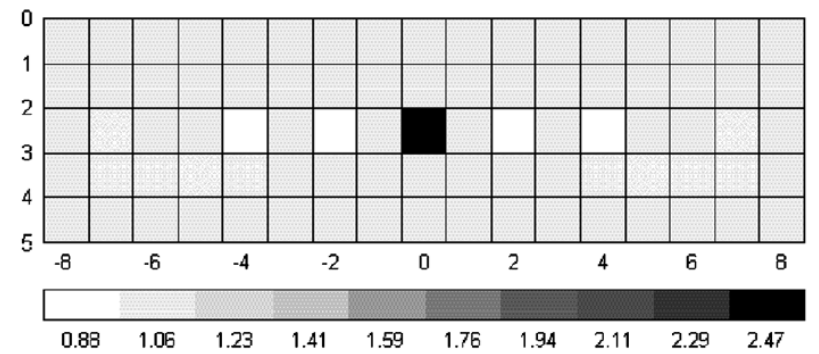

d)

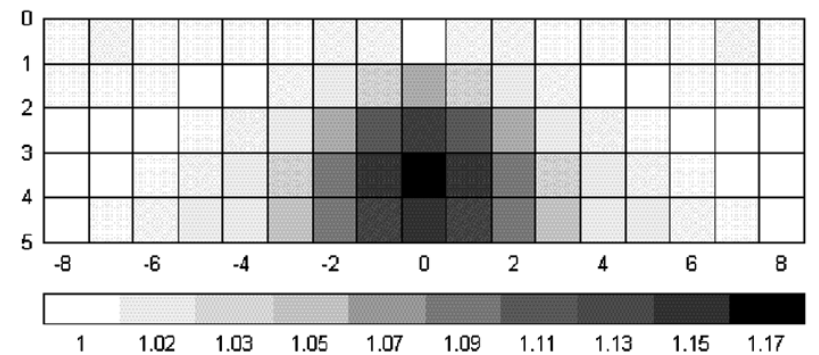

Fig. 9. Two-dimensional images obtained by the dipole-dipole array for a perfectly conducting sphere of 0.5 unit radius placed at $x=y=0, z=2.5$, and the five image-reconstruction algorithms considered. (a) Marquardt $\left(\lambda=2.85 \times 10^{-12}\right)$. (b) TSVD $(r=50)$. (c) Occam $\left(\lambda=3.07 \times 10^{-12}\right)$. (d) Total backprojection. (e) Equipotential backprojection.

selected $k=10$, based on experimental results. The 2-D images obtained correspond to a vertical slice just under the electrode array. Fig. 6 shows a slice divided in 85 cubic voxels (17 in the horizontal plane and five in the vertical plane) of side dimension 1 (arbitrary unit). We used 16 equally spaced electrodes separated 1 unit. Electrode 1 was at $x=-7.5, y=0, z=0$, and electrode 16 was at $x=7.5, y=0, z=0$. The origin of coordinates was between electrodes 8 and 9 .

Fig. 7 shows the respective 2-D images obtained with the five algorithms for a perfectly conducting sphere of 0.5 unit radius placed at $x=y=0$ at a depth of 1.5 units, for the two electrode arrays. The bottom scale in each image represents conductivities normalized to the homogeneous medium. The least-squares methods considered outperform the bakcprojection methods, whose images are blurred and depend on the electrode array used. The Occam method smoothes the image and elongates the target in the vertical direction. Marquardt and TSVD methods yield the best performance and perfectly locate the position of the target for both electrode arrays. The voxel corresponding to the target shows an increase in conductivity. For the Marquardt and Occam methods, we selected $\lambda=10 c$ ( $c$ being the value determined by the L-curve method). For the TSVD method, we selected $\mathrm{r}$ (rank of the inverse matrix $)=35$.
Fig. 8 shows the L-curve obtained with the dipole-dipole array for, respectively, the Marquardt, TSVD, and Occam methods. The results with the Schlumberger array were very similar and are not shown here. For the TSVD method, Fig. 8(b) shows the discrete L-curve as a function of the rank $r$ of the inverse matrix.

Fig. 9 shows images obtained when using the dipole-dipole electrode array for the same conducting sphere in Fig. 7 placed at $x=y=0, z=2.5$. Because a deeper sphere produces a smaller change in the mutual impedance, $\Delta \boldsymbol{Z}$, to obtain a good estimate of the true solution $\lambda$ must be decreased in the Marquardt and Occam methods, and the rank $r$ must be increased in the TSVD method. In fact, the smaller singular values of $\Lambda$ in (14) correspond to the deeper voxels of the model [9]. In Fig. 9, we selected $\lambda=100 c$ (resulting in $\lambda$ values three to four orders of magnitude smaller than those used in Fig. 7) for the Marquardt and Occam methods, and $r=50$ for the TSVD method. Least-squares methods yielded a conductivity for the target similar to that in Fig. 7, but backprojection methods obtained a smaller conductivity for deeper immersion. The modified Schlumberger array (not shown) yielded similar results when using least-squares methods, but backprojection methods obtained a different conductivity and depth for the sphere. 
TABLE I

NCE FOR THE FIVE ALGORITHMS USED AND FOR THE SCHLUMBERGER AND DipOLE-DipOLE ELECTRODE CONFIGURATIONS

\begin{tabular}{|c|c|c|c|c|}
\hline \multirow{2}{*}{} & \multicolumn{2}{|c|}{ Schlumberger } & \multicolumn{2}{c|}{ Dipole-dipole } \\
\cline { 2 - 5 } & $x=y=0, z=1.5$ & $x=y=0, z=2.5$ & $x=y=0, z=1.5$ & $x=y=0, z=2.5$ \\
\hline Marquardt & $2.40 \times 10^{-2}$ & $2.91 \times 10^{-2}$ & $2.08 \times 10^{-2}$ & $3.00 \times 10^{-2}$ \\
\hline TSVD & $2.27 \times 10^{-2}$ & $3.58 \times 10^{-2}$ & $1.43 \times 10^{-2}$ & $2.80 \times 10^{-2}$ \\
\hline Occam & 0.105 & $6.12 \times 10^{-2}$ & $7.39 \times 10^{-2}$ & $6.25 \times 10^{-2}$ \\
\hline Total backprojection & 0.226 & 0.373 & 0.248 & 0.308 \\
\hline Equip. backprojection & 0.302 & 0.349 & 0.234 & 0.282 \\
\hline
\end{tabular}

a)

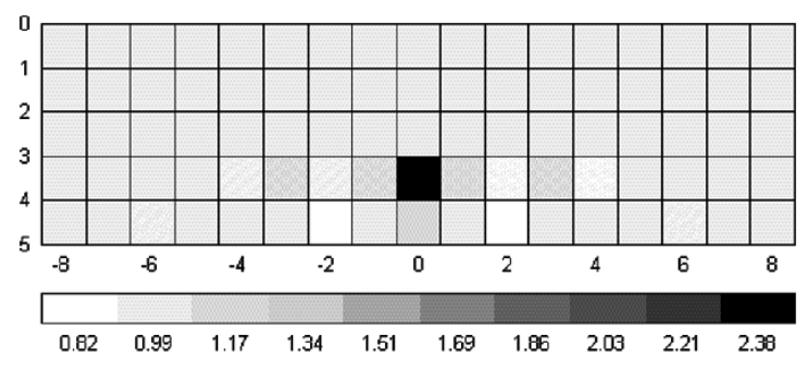

b)

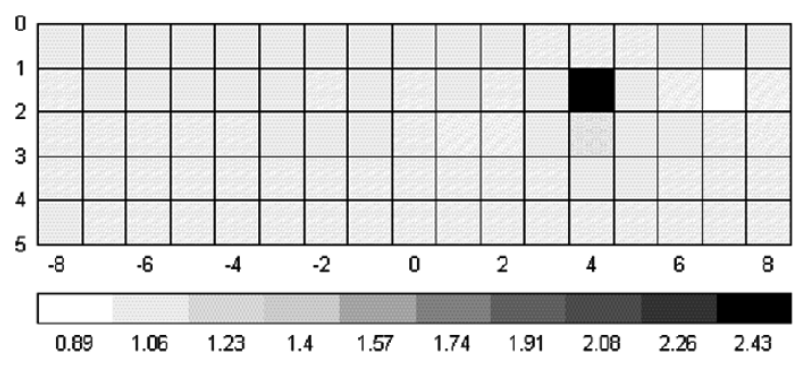

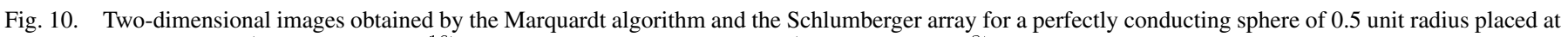
(a) $x=y=0, z=3.5\left(\lambda=1.92 \times 10^{-16}\right)$, and (b) $x=4, y=0, z=1.5\left(\lambda=2.45 \times 10^{-8}\right)$.

In order to more objectively compare the performance of the five algorithms used we can define a normalized conductivity error (NCE) as

$$
\mathrm{NCE}=\sqrt{\frac{1}{Q} \sum_{j=1}^{Q}\left(\frac{\sigma_{j}^{\text {est }}}{\max \left(\sigma_{j}^{\text {est }}\right)}-\frac{\sigma_{j}^{\text {ideal }}}{\max \left(\sigma_{j}^{\text {ideal }}\right)}\right)^{2}}
$$

where $\sigma_{j}^{\text {est }}$ is the conductivity estimated by the algorithms and $\sigma_{j}^{\text {ideal }}=1$ for $j=26$ in Fig. 7 and for $j=43$ in Fig. 9, and $\sigma_{j}^{j \text { ideal }}=0$ in the rest of pixels. Table I shows the NCE value for the cases in Figs. 7 and 9, including the results of the modified Schlumberger array not shown in those figures. These numerical values agree with the qualitative conclusions presented before. Backprojection algorithms present a larger error in all cases. Marquardt and TSVD show the smallest errors. Because the TSVD method required the use of a specialized Toolbox of MATLAB in order to automatically determine the rank $r$ of $S$, we decided to use the Marquardt method in the remaining images, including those from experimental results.

Fig. 10 shows images obtained with the modified Schlumberger array and the Marquardt method for a conductive sphere placed at $x=y=0, z=3.5$ (hence, deeper than in Figs. 8 and 9), and $x=4, y=0, z=1.5$. The sphere position was correctly detected in both cases. The value of $\lambda$ in Fig. 10(a) was four orders of magnitude smaller than in Fig. 9. Section V shows that, when using experimental data, the value of $\lambda$ cannot be decreased that much because of (unavoidable) measurement errors. Hence, images for deeper objects should blur.

\section{EXPERIMENTAL SETUP}

Experimental data were obtained from a plastic water tank $(40 \times 35 \times 20 \mathrm{~cm})$ (Fig. 11). We used 16 stainless steel electrodes of $3 \mathrm{~mm}$ in diameter, uniformly spaced $2 \mathrm{~cm}$ (one unit). The targets were a metallic (conductive) sphere of radius

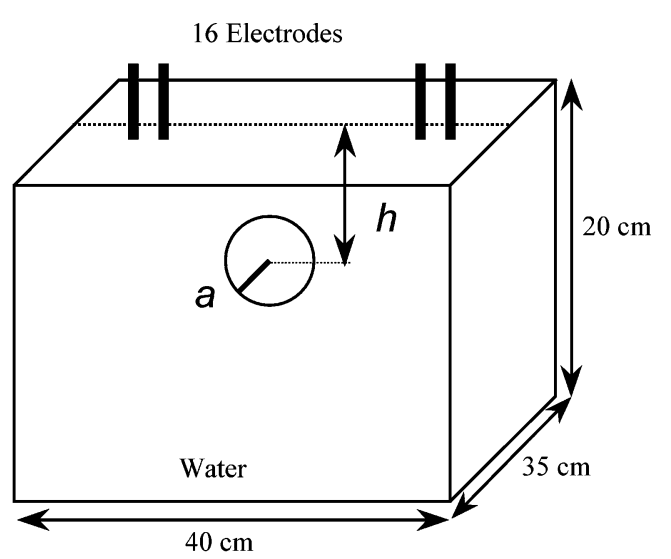

Fig. 11. Plastic water tank $(40 \times 35 \times 20 \mathrm{~cm})$ used to obtain experimental data. Targets were a rubber ball of radius $2.2 \mathrm{~cm}$ (1.1 units) and a conducting sphere of radius $1.75 \mathrm{~cm}$ (0.875 units).

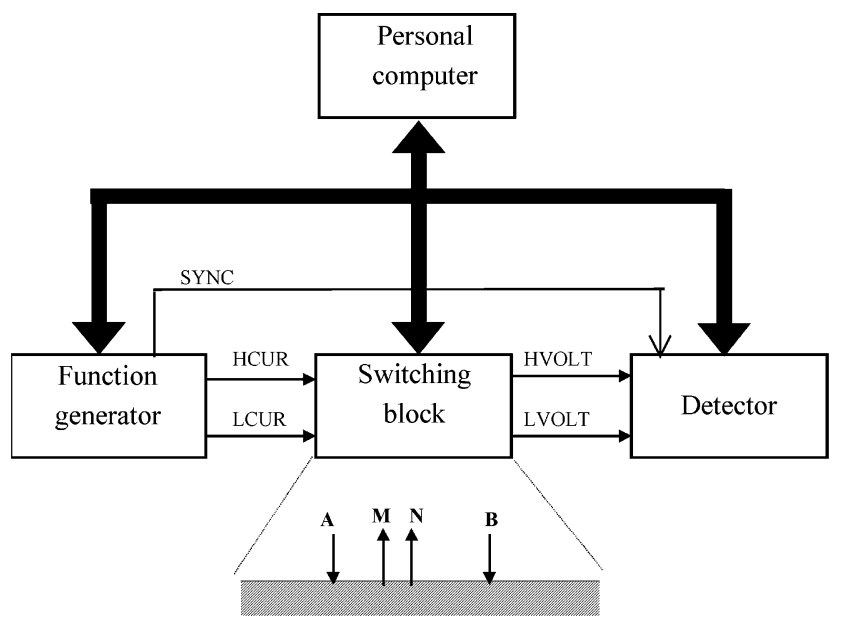

Fig. 12. Block diagram of the computer-controlled data acquisition system. 
a)

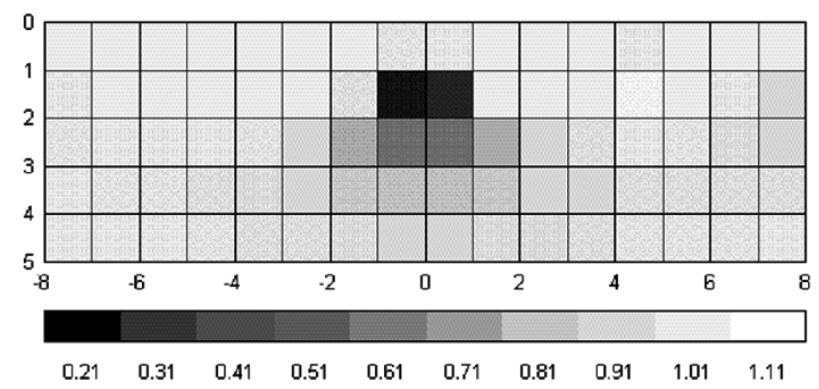

b)

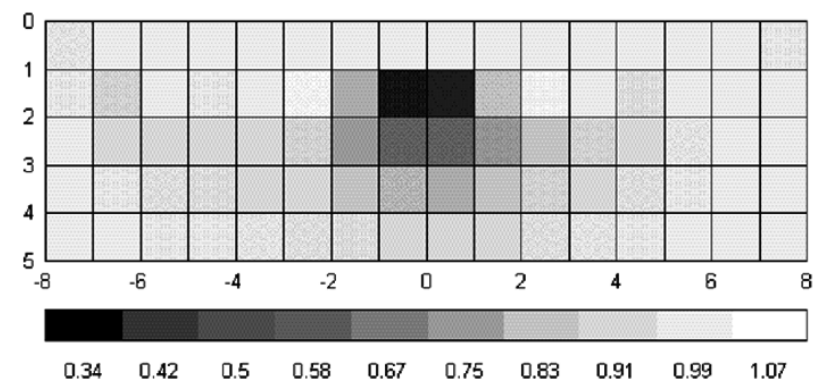

Fig. 13. Two-dimensional images obtained by the Marquardt algorithm from experimental data for the rubber ball placed at $x=y=0$, at a depth of 4 cm with (a) the Schlumberger array $\left(\lambda=9.19 \times 10^{-4}\right)$ and $(b)$ the dipole-dipole array $\left(\lambda=1.99 \times 10^{-2}\right)$. The 2 -D model consists of 80 voxels $(16 \times 5)$ of dimensions $1 \times 2 \times 1$ units.

a)

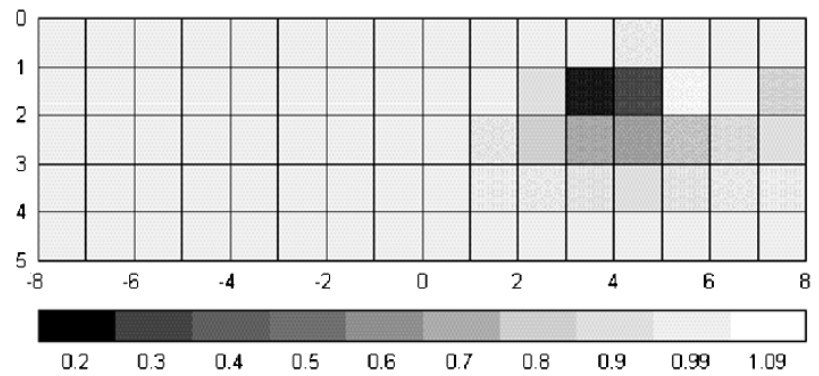

b)

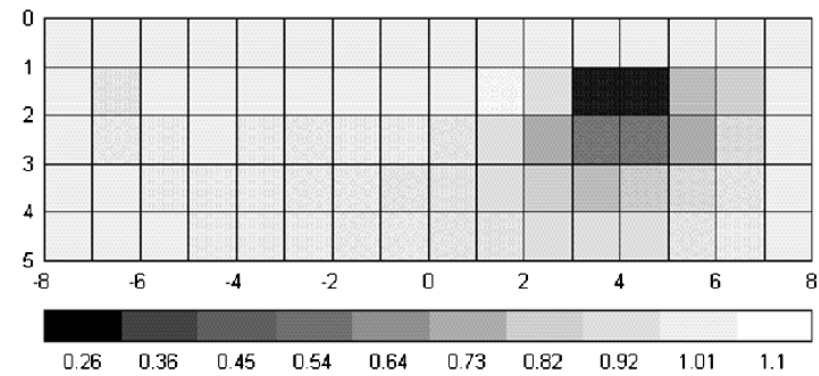

Fig. 14. Two-dimensional images obtained for the rubber ball shifted by $8 \mathrm{~cm}$ to the right at a depth of $4 \mathrm{~cm}$ when using (a) the Schlumberger array $(\lambda=$ $\left.4.47 \times 10^{-4}\right)$ and (b) the dipole-dipole array $\left(\lambda=9.04 \times 10^{-3}\right)$.

$1.75 \mathrm{~cm}(0.875$ units) and a rubber (nonconductive) ball of radius $2.2 \mathrm{~cm}$ (1.1 unit).

Fig. 12 shows the block diagram of the computer-controlled data-acquisition system. The signal generator injected an ac current between electrodes $\mathrm{A}$ and $\mathrm{B}$, the detector measured the voltage difference between electrodes $\mathrm{M}$ and $\mathrm{N}$, and the switching block selected the injecting and detecting electrodes for each measurement. A personal computer controlled the current injection, voltage detection, and electrode switching via standard computer buses. Injecting an ac current avoids redox reactions, which can degrade the electrodes, and dc voltages attributable to electrochemical potentials, and yields small electrode impedances, which help in reducing measurement errors.

The ratio between the largest and the smallest predicted voltages is, respectively, 28 and 690 for the modified Schlumberger and the dipole-dipole arrays [9]. This resulted in a larger signal-to-noise ratio (SNR) for the modified Schlumberger array, especially for the smallest voltage measurements.

\section{IMAGES FROM EXPERIMENTAL DATA}

Images from experimental data were obtained by using the Marquardt-Levenberg method and the model in Fig. 6 with voxels of dimensions $1 \times 2 \times 1$ units. To reduce the effects of the finite dimensions of the tank, we used both a reference set of measurements taken before immersing the target, and a normalized version of the reconstruction algorithm. This procedures also reduced errors due to the inaccuracy in the position of the electrodes [40]. The normalized algorithm was

$$
\Delta \boldsymbol{\Sigma}_{\text {rel }}=\left(S_{\text {rel }}^{T} S_{\text {rel }}+\lambda I\right)^{-1} S_{\text {rel }}^{T} \Delta Z_{\text {rel }} .
$$

The elements of $\Delta \boldsymbol{\Sigma}_{\mathrm{rel}}, \Delta \boldsymbol{Z}_{\mathrm{rel}}$ and $S_{\text {rel }}$ were

$$
\begin{aligned}
\Delta \sigma_{j, \mathrm{rel}} & =\frac{\Delta \sigma_{j}}{\sigma^{0}} \quad j=1 \ldots P \\
\Delta z_{i, \mathrm{rel}} & =\frac{\Delta z_{i}}{z_{i}^{0}}=\frac{z_{i}-z_{i}^{0}}{z_{i}^{0}} \quad i=1 \ldots Q \\
s_{i j, \mathrm{rel}} & =\frac{\sigma^{0}}{z_{i}^{0, t}} s_{i j}
\end{aligned}
$$

where $\sigma^{0}$ was the homogeneous conductivity, $z$ and $z^{0}$ were the experimental data measured with and without the target, and $z^{0, t}$ was the theoretical mutual impedance calculated for a homogeneous medium. In some reconstructions algorithms, $s_{i j, \text { rel }}$ is obtained by dividing each $s_{i j}$ by the sum $\sum s_{j}$, but this can lead to unacceptable results.

Fig. 13 shows 2-D images obtained with each electrode array for the rubber ball placed at $x=y=0$ at a 4-cm depth (two units). The bottom scale in the image represents conductivities normalized to the homogeneous medium $(0.15 \mathrm{~S} / \mathrm{m}$ approximately). Both arrays correctly located the target as a conductivity decrease in the corresponding pixels. $\lambda$ was larger for the dipole-dipole configuration, probably because of the smaller SNR for the corresponding measurements. Fig. 14 shows 2-D images obtained for the ball when shifted by $8 \mathrm{~cm}$ (four units) to the right. Both electrode configurations correctly located the target. Similar results were obtained when the ball was shifted to the left. 


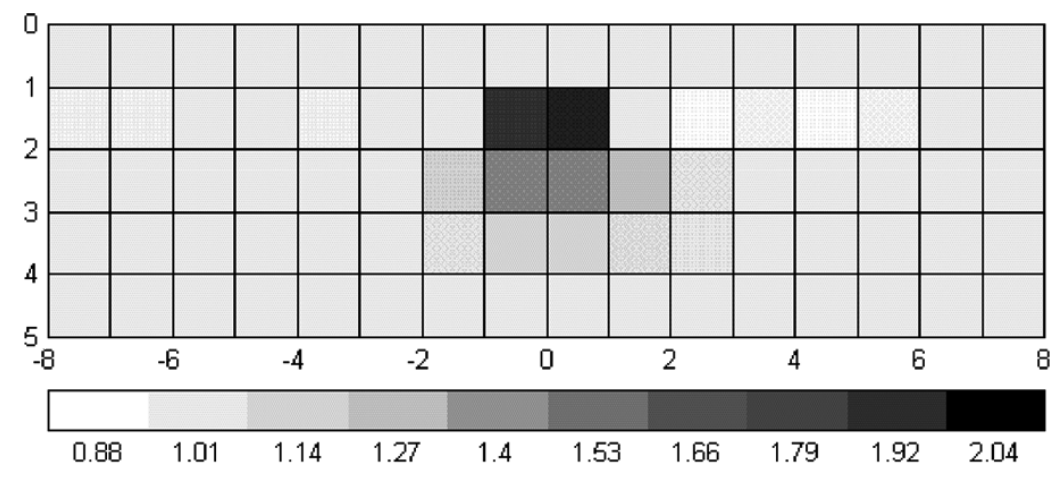

Fig. 15. Two-dimensional images obtained for the conducting sphere immersed at $x=0, y=0, z=4 \mathrm{~cm}\left(\lambda=1.12 \times 10^{-3}\right)$.

a)

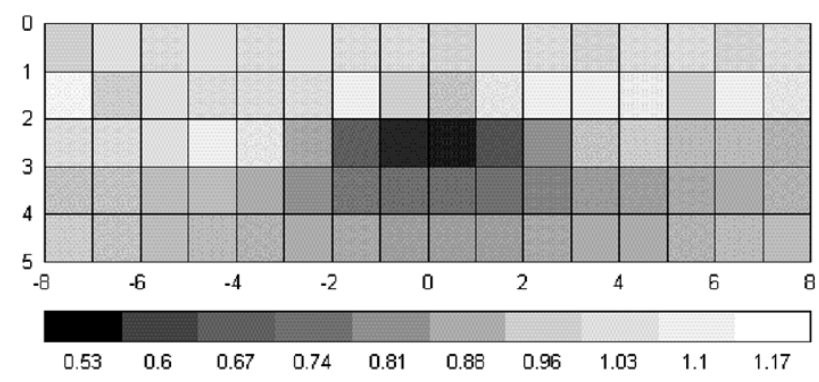

b)

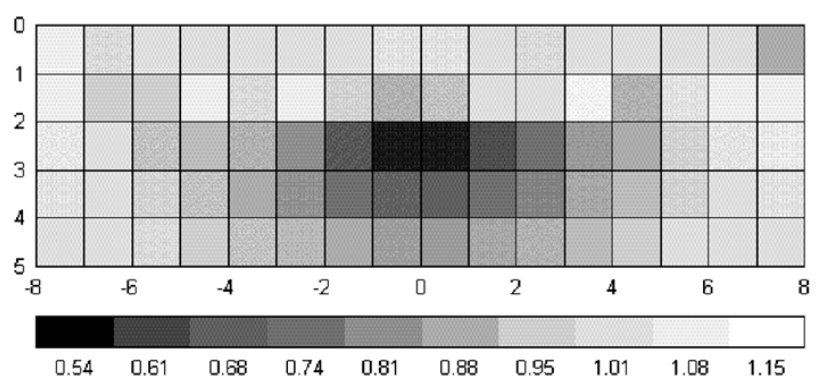

Fig. 16. Two-dimensional images obtained for the rubber ball placed at $x=y=0$, at a depth of $6 \mathrm{~cm}$, with (a) Schlumberger $\left(\lambda=9.19 \times 10^{-5}\right)$ and (b) dipole-dipole $\left(\lambda=6.55 \times 10^{-3}\right)$ electrode arrays.

a)

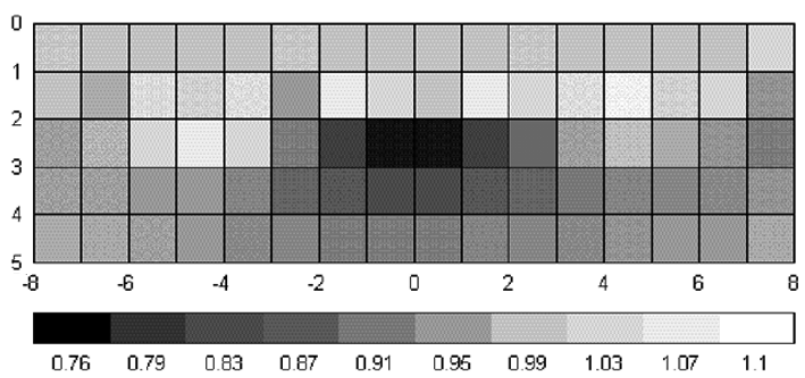

b)

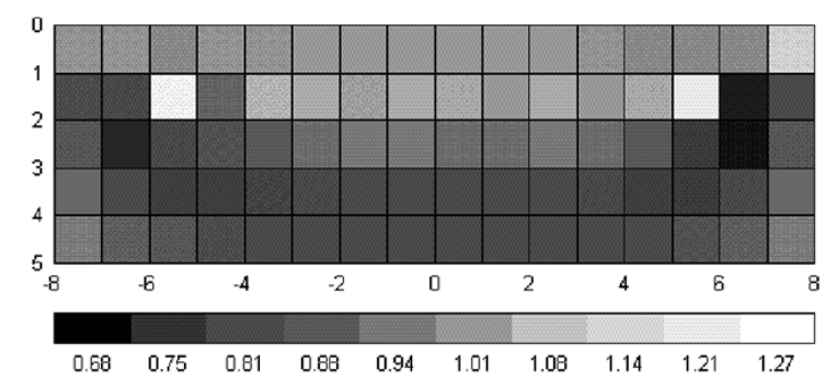

Fig. 17. Two-dimensional images obtained for the rubber ball placed at $x=y=0$, at a depth of $8 \mathrm{~cm}$, with (a) Schlumberger $\left(\lambda=1.43 \times 10^{-4}\right)$ and (b) dipole-dipole $\left(\lambda=4.31 \times 10^{-3}\right)$ electrode arrays.

Fig. 15 shows the image obtained by the Schlumberger electrode array for the metallic sphere when placed at $x=y=0$ at 4-cm depth (two units). The target was correctly located and the corresponding pixels showed an increased conductivity.

Fig. 16 shows images obtained with each electrode array for the rubber ball placed at $x=y=0$ at a 6 -cm depth. The target was poorly identified, probably because of the relative larger effect that measurement nonidealities had on the smaller $\Delta \boldsymbol{Z}$. Some nonidealities were voltage measurement errors, the finite dimensions of the tank, and errors in electrode positioning. Furthermore, the conductivity change was smaller than in Fig. 13 because the target was ascribed to more pixels.

Fig. 17 shows images obtained by each electrode array for the rubber ball placed at a $8-\mathrm{cm}$ depth. The Schlumberger array incorrectly located the target at a $6-\mathrm{cm}$ depth. The image from the dipole-dipole array was severely distorted, probably because of the lower SNR in the measurements.
Fig. 18 shows images obtained with the dipole-dipole configuration for the rubber ball placed at $x=y=0$ at depths of 6 and $8 \mathrm{~cm}$, when neglecting measurements whose SNR was smaller than $70 \mathrm{~dB}$. The images outperformed those obtained in Figs. 16 and 17, and the target was correctly identified in both cases. The value of $\lambda$ used was much smaller than that used in Figs. 16 and 17 for the dipole-dipole configuration, thanks to the elimination of the "worst" measurements. For the Schlumberger array, all the measurement had SNR $>70 \mathrm{~dB}$, so that we could not obtain any further improvement. The dipole-dipole array outperformed the Schlumberger array probably because of the better conditioning of the sensitivity matrix [9].

Finally, Fig. 19 shows images obtained with the Schlumberger array when the two spheres were simultaneously immersed in the water tank: the conducting sphere at $x=-4 \mathrm{~cm}, y=0, z=4 \mathrm{~cm}$, and the rubber ball at $x=4 \mathrm{~cm}$, $y=0, z=4 \mathrm{~cm}$. Both targets were correctly located and 
a)

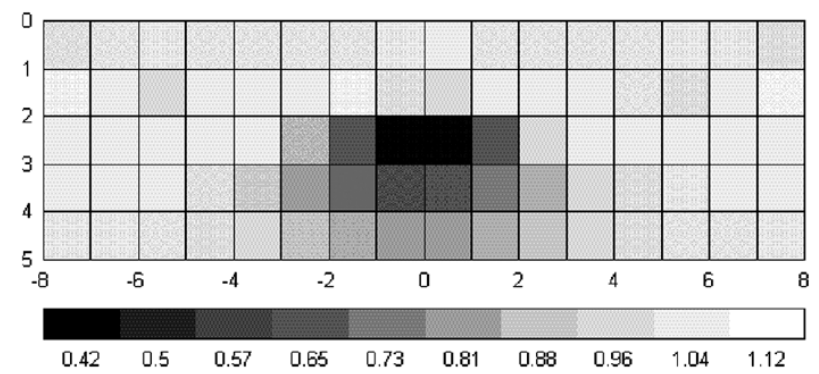

b)

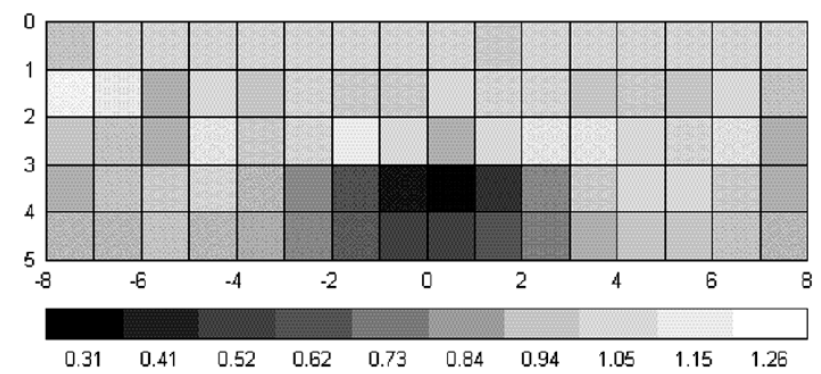

Fig. 18. Two-dimensional images obtained with the dipole-dipole electrode array, using only the data with SNR $>70 \mathrm{~dB}$, for the rubber ball placed at $x=$ $y=0$, at a depth of (a) $6 \mathrm{~cm}\left(\lambda=1.37 \times 10^{-4}\right)$ and (b) $8 \mathrm{~cm}\left(\lambda=2.23 \times 10^{-5}\right)$.

a)

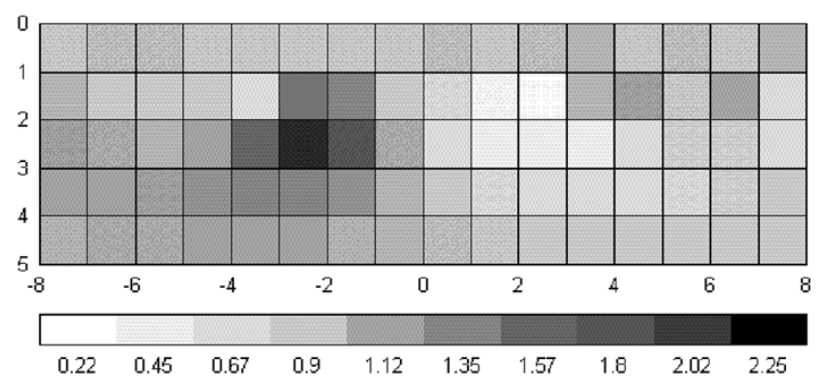

b)

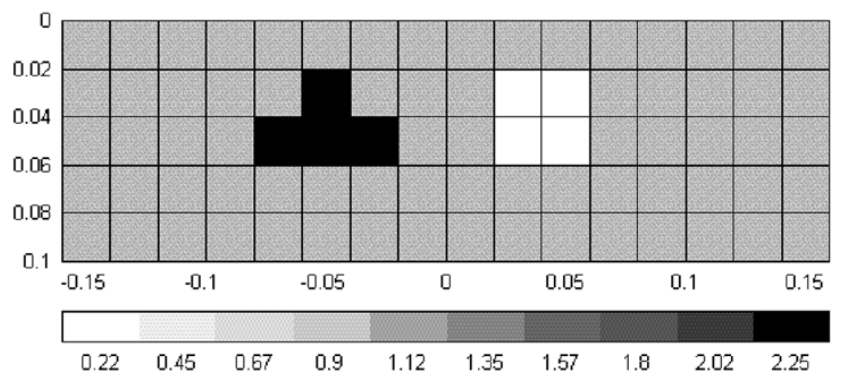

Fig. 19. Two-dimensional images obtained by the Schlumberger electrode array $\left(\lambda=1.74 \times 10^{-4}\right)$ for the conducting sphere and the rubber ball simultaneously immersed at a 4-cm depth and, respectively, $x=-4 \mathrm{~cm}$ and $x=4 \mathrm{~cm}$. (a) Direct image. (b) Processed image.

the respective conductivity values were similar to those obtained when the targets were separately immersed. We further processed the image to improve their distinguishability. In Fig. 19(b), for example, conductivity values smaller than 0.55 were equated to the minimal conductivity (0.22), and conductivity values larger than 1.45 were equated to the maximal conductivity (2.25). The remaining conductivity values were equated to the normalized homogeneous value (1.0). The distinguishability clearly improved.

The Marquardt algorithm showed in (24) has also been successfully employed for detecting leaks in a buried PVC tube [9].

\section{CONCLUSION}

We have compared five different noniterative algorithms for electrical resistivity imaging applied to subsurface local anomalies. Three of the algorithms were least-squares based and two algorithms applied backprojection. We have shown that least-squares methods are theoretically sounder than backprojection algorithms. We have compared the performance of the five algorithms using synthetic data obtained from an analytical solution, when using two different electrode arrays: Schlumberger and dipole-dipole. Images obtained with backprojection algorithms were blurred and depended on the electrode array used. Furthermore, the conductivity changes obtained decreased as the depth of the target increased. On the contrary, least-squares methods, specially Marquardt and TSVD, perfectly located the target without depending on the electrode array used. The regularization parameter $\lambda$ has been estimated by using the L-curve method, with excellent results.
This eliminated the overburdening task of choosing $\lambda$ by a trial-and-error procedure.

Experimental data were obtained from a plastic water tank wherein conductive and nonconductive targets were immersed. A computer-controlled data-acquisition system was used together with a 16 equally spaced electrode array. Both Schlumberger and dipole-dipole electrode arrays were implemented. To reduce the effects of the finite dimension of the tank and the inaccuracy in the position of the electrodes, a reference set of measurements was taken before immersing the target and a normalized version for the Marquardt algorithm was used. The images deteriorated for deeper targets, specially with the dipole-dipole array. However, eliminating measurements with the lowest SNR improved the images and allowed the detection of the target even when immersed at a depth of about four times the target diameter.

Some important aspects that should be addressed in future are the effects of off-axis targets into 2-D images, the reconstruction of truly 3-D images, the in-depth study of the sensitivity matrix, and the analysis of noise and interference in measurements and their effect upon the reconstructed images.

\section{REFERENCES}

[1] M. H. Loke and R. D. Barker, "Practical techniques for 3D resistivity surveys and data inversion," Geophys. Prospect., vol. 44, pp. 499-523, 1996.

[2] D. W. Oldenburg, P. R. McGillivray, and R. G. Ellis, "Generalized subspace methods for large-scale inverse problems," Geophys. J. Int., vol. 114, pp. 12-20, 1993.

[3] M. H. Loke and R. D. Barker, "Least-squares deconvolution of apparent resistivity pseudosections," Geophysics, vol. 60, no. 6, pp. 1682-1690, 1995. 
[4] C. J. Kotre, "Subsurface electrical impedance imaging using orthogonal linear electrode arrays," Proc. Inst. Elect. Eng., Sci. Meas. Tech., vol. 143, no. 1, pp. 41-46, 1996.

[5] - "Subsurface electrical impedance imaging: Measurement strategy, image reconstruction and in vivo results," Physiol. Meas. A, vol. 17, pp. 197-204, 1996.

[6] J. L. Mueller, D. Isaacson, and J. C. Newell, "Reconstruction of conductivity changes due to ventilation and perfusion from EIT data collected on a rectangular electrode array," Physiol. Meas., vol. 22, no. 1, pp. 97-106, Feb. 2001

[7] M. Noel and B. Xu, "Archaeological investigation by electrical resistivity tomography: A preliminary study," Geophys. J. Int., vol. 107, pp. 95-102, 1991.

[8] J. Jordana, M. Gasulla, and R. Pallas-Areny, "Electrical resistance tomography to detect leaks from buried pipes," Meas. Sci. Technol., vol. 12, pp. 1061-1068, 2001.

[9] M. Gasulla, "Images of the electrical impedance distribution of the subsoil applied to the detection of local objects," Ph.D. dissertation, Dept. Electron. Eng., Tech. Univ. Catalonia, Barcelona, Spain, 1999. (in Spanish).

[10] M. Gasulla, J. Jordana, and R. Pallas-Areny, "2D and 3D subsurface resistivity imaging using a constrained least-squares algorithm," presented at the 1st World Congr. Industrial Process Tomography, Apr. 1999.

[11] D. B. Geselowitz, "An application of electrocardiographic lead theory to impedance plethysmography," IEEE Trans. Biomed. Eng., vol. BE-18, no. 1, pp. 38-41, Jan. 1971.

[12] J. Lehr, "A vector derivation useful in impedance plethysmographic field calculations," IEEE Trans. Biomed. Eng., vol. BE-19, no. 3, pp. 156-157, Mar. 1972.

[13] T. Murai and Y. Kagawa, "Electrical impedance computed tomography based on a finite element model," IEEE Trans. Biomed. Eng., vol. BE-32, no. 3, pp. 177-184, Mar. 1985.

[14] L. R. Lines and S. Treitel, "Tutorial. A review of least squares inversion and its application to geophysical problems," Geophys. Prospect., vol. 32, pp. 159-186, 1984

[15] G. H. Golub and C. F. Van Loan, Matrix Computations, 2nd ed. Baltimore, MD: The Johns Hopkins Univ. Press, 1989.

[16] W. Menke, Geophysical Data Analysis: Discrete Inverse Theory, 2nd ed. San Diego, CA: Academic, 1989.

[17] C. Hansen, "Regularization tools: A Matlab package for analysis and soluton of discrete ill-posed problems," Danish Comput. Center Res. Ed., Technical Univ. Denmark, 1992.

[18] N. J. Avis and D. C. Barber, "Image reconstruction using nonadjacent drive configurations," Physiol. Meas., vol. 15, pp. 153-160, 1994.

[19] — "Incorporating a priori information into the Sheffield filtered backprojection algorithm," Physiol. Meas. A, vol. 16, pp. 111-122, 1995.

[20] B. M. Eyuboglu, "An interleaved drive electrical impedance tomography image recontruction algorithm," Physiol. Meas. A, vol. 17, pp. 59-71, 1996.

[21] F. Kleinermann, N. J. Avis, S. K. Judah, and D. C. Barber, "Threee-dimensional image reconstruction for electrical impedance tomography," Physiol. Meas. A, vol. 17, pp. 77-83, 1996.

[22] S. Meeson, A. L. T. Killingback, B. H. Blott, C. Mitchell, D. F. Evans, and P. J. Milla, "Optimal filtering of EIT data in espectral expansion analysis," Physiol. Meas. A, vol. 17, pp. 85-90, 1996.

[23] W. Q. Yang and L. Peng, "Image reconstruction algorithms for electrical capacitance tomography," Meas. Sci. Technol., vol. 14, pp. R1-R13, 2003.

[24] A. N. Tikhonov and V. Y. Arsenin, Solutions of Ill-Posed Problems. Washington, DC: Winston, 1977.

[25] P. Hua, J. G. Webster, and W. J. Tompkins, "A regularised electrical impedance tomography reconstruction algorithm," Clin. Phys. Physiol. Meas. A, vol. 9, pp. 137-141, 1988.

[26] Y. Sasaki, "Resolution of resistivity tomography inferred from numerical simulation," Geophys. Prospect., vol. 40, pp. 453-463, 1992.

[27] C. Hansen and D. P. O'Leary, "Analysis of discrete ill-posed problems by means of the L-curve," SIAM Rev., vol. 34, no. 4, pp. 561-580, 1992.

[28] - "The use of the L-curve in the regularization of discrete ill-posed problems," SIAM J. Sci. Comput., vol. 14, no. 6, pp. 1487-1503, 1993.

[29] D. J. LaBrecque, M. Miletto, W. Daily, A. Ramirez, and E. Owen, "The effects of noise on Occam's inversion of resistivity tomography data," Geophysics, vol. 61, no. 2, pp. 538-548, 1996.

[30] M. H. Loke and R. D. Barker, "Rapid least-squares inversion of apparent resistivity pseudosections by a quasi-Newton method," Geophys. Prospect., vol. 44, pp. 131-152, 1996.
[31] Y. Sasaki, "3-D resistivity inversion using the finite-element method," Geophysics, vol. 59, no. 11, pp. 1839-1848, 1994.

[32] W. R. Breckon and M. K. Pidock, "Data errors and reconstruction algorithms in electrical impedance tomography," Clin. Phys. Physiol. Meas. A, vol. 9, pp. 105-109, 1988.

[33] M. H. Loke and T. Dahlin, "A comparison of different least-squares formulations for the inversion of resistivity and IP data," in Proc. IV Meeting of the Environmental and Engineering Geophysical Society, Barcelona, Spain, Sep. 1998, pp. 825-828.

[34] C. J. Kotre, "Detection of sub-surface objects by electrical impedance tomography," in Proc. EUREL Int. Conf. Detection of Abandoned Land Mines, Edinburgh, U.K., Oct. 1996, pp. 67-71.

[35] P. Tsourlos, J. Szymanski, J. Dittmer, and G. Tsokas, "The use of weighted back-projection for fast inversion of vertical profiling resistivity data," presented at the 2nd Congr. Hellenic Geophysical Union, Florina, Greece, May 1993.

[36] D. C. Barber, B. H. Brown, and I. L. Freeston, "Imaging spatial distributions of resistivity using applied potential tomography," Electron. Lett., vol. 19 , no. 22, pp. 933-935, 1983.

[37] D. C. Barber and B. H. Brown, "Applied potential tomography," J. Phys. E: Sci. Instrum., vol. 17, pp. 723-733, 1984.

[38] H. M. Powell, D. C. Barber, and I. L. Freeston, "Impedance imaging using linear electrode arrays," Clin. Phys. Physiol. Meas. A, vol. 8, pp. 109-118, 1987.

[39] W. M. Telford, L. P. Geldart, and R. E. Sheriff, Applied Geophysics, 2nd ed. New York: Cambridge Univ. Press, 1990.

[40] D. C. Barber and B. H. Brown, "Errors in reconstruction of resistivity images using a linear reconstruction technique," Clin. Phys. Physiol. Meas. A, vol. 9, pp. 101-104, 1988.

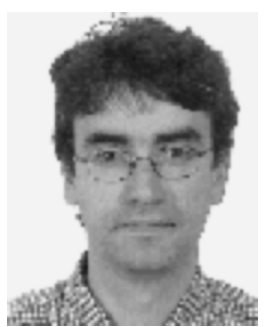

Manel Gasulla was born in Vinaròs, Spain, on May 26, 1967. He received the M.Sc. and Ph.D. degrees in telecommunication engineering from the Technical University of Catalonia (UPC), Barcelona, Spain, in 1992 and 1999, respectively.

Since 1993, he has been with the UPC, where he is an Associate Professor, engaged in teaching analog electronics and electronic instrumentation. From 2001 to 2002, he was a Visiting Postdoctorate at the Electronic Instrumentation Laboratory, Delft University of Technology, Delft, The Netherlands. His research interests include smart capacitive sensors systems, energy harvesting methods, autonomous sensors, electrical impedance measurements, and subsurface resistivity imaging.

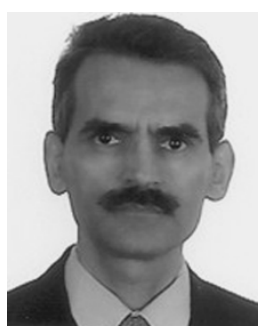

Ramon Pallàs-Areny (M'81-SM'88-F'98) received the ingeniero industrial and doctor ingeniero industrial degrees from the Technical University of Catalonia (UPC), Barcelona, Spain, in 1975 and 1982, respectively.

In 1989 and 1990, he was a visiting Fulbright Scholar, and in 1997 and 1998, he was an Honorary Fellow at the University of Wisconsin, Madison. In 2001, he was nominated Professor Honoris Causa by the Faculty of Electrical Engineering of the University of Cluj-Napoca, Romania. He is a Professor of electronic engineering with the UPC and teaches courses in medical and electronic instrumentation. His research interests include instrumentation methods and sensors based on electrical impedance measurements, sensor interfaces, noninvasive physiological measurements, and electromagnetic compatibility in electronic systems. He is the author of several books on instrumentation in Spanish and Catalan, the latest one being Sensors and Interfaces, Solved Problems (Barcelona, Spain: Edicions UPC, 1999). He is also coauthor (with John G. Webster) of Sensors and Signal Conditioning, 2nd ed. (New York: Wiley, 2001) and Analog Signal Processing (New York: Wiley, 1999).

Dr. Pallàs-Areny was a recipient, with John G. Webster, of the 1991 Andrew R. Chi Prize Paper Award from the IEEE Instrumentation and Measurement Society. In 2000, he received the Award for Quality in Teaching granted by the Board of Trustees of the UPC, and in 2002 the Narcís Monturiol Medal from the Autonomous Government of Catalonia. He is a member of the International Society for Measurement and Control (ISA). 